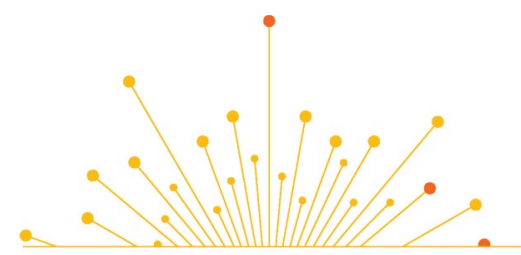

SCIENCE TABLE

COVID-19 ADVISORY FOR ONTARIO

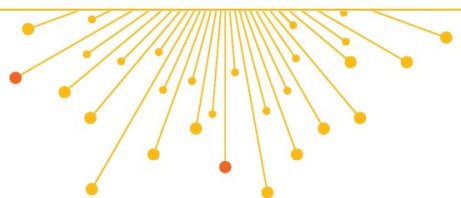

Version 1.0

Published: March 17, 2021

Citation: Huynh T, Sava N, Hahn-Goldberg S, et al. Mobile on-site COVID-19 vaccination of naturally occurring retirement communities by neighbourhood risk in Toronto. Science Briefs of the Ontario COVID-19 Science Advisory Table. 2021;2(14). https:// doi.org/10.47326/ocsat.2021.02.14.1.0

Author Affiliations: The affiliations of the members of the Ontario COVID-19 Science Advisory Table can be found at https:// covid19-sciencetable.ca/.

Declarations of Interest: The declarations of interest of the members of the Ontario COVID-19 Science Advisory Table can be found at https://covid19-sciencetable.ca/.

About Us: The Ontario COVID-19 Science Advisory Table is a group of scientific experts and health system leaders who evaluate and report on emerging evidence relevant to the COVID-19 pandemic, to inform Ontario's response. Our mandate is to provide weekly summaries of relevant scientific evidence for the COVID-19 Health Coordination Table of the Province of Ontario, integrating information from existing scientific tables, Ontario's universities and agencies, and the best global evidence. The Science Table summarizes its findings for the Health Coordination Table and the public in Science Briefs.

Correspondence to: Secretariat of the Ontario COVID-19 Science Advisory Table (info@covid19-sciencetable.ca)

Copyright: 2021 Ontario COVID-19 Science Advisory Table. This is an open access document distributed under the terms of the Creative Commons Attribution License, which permits unrestricted use, distribution, and reproduction in any medium, provided that the original work is properly cited.

The views and findings expressed in this Science Brief are those of the authors and do not necessarily reflect the views of all of the members of the Ontario COVID-19 Science Advisory Table, its Working Groups, and its partners.

\section{Mobile On-Site COVID-19 Vaccination of Naturally Occurring Retirement Communities by Neighbourhood Risk in Toronto}

Tai Huynh, Nathalie Sava, Shoshana Hahn-Goldberg, Jen Recknagel, Isaac I. Bogoch, Kevin A. Brown, Vinita Dubey, Shiran Isaacksz, Peter Jüni, Fiona G. Kouyoumdjian, Antonina Maltsev, Douglas G. Manuel, Danielle Martin, Josh Matlow, Allison McGeer, Christa S. Mills, Elizabeth Niedra, Jeff Powis, Paula A. Rochon, Samir K. Sinha, Nathan M. Stall, Adalsteinn D. Brown on behalf of the Ontario COVID-19 Science Advisory Table

\section{Key Message}

Naturally occurring retirement communities (NORCs) are apartment, condo, co-op and social housing buildings that while not purpose-built for older adults, have become home to a high number of them.

In Toronto, there are 489 residential buildings that are NORCs. Of these, 256 are located in neighbourhoods with the highest cumulative incidence of SARS-CoV-2, and are home to 40,955 older adults 65 years of age and above, including 18,144 older adults 80 years of age and above.

Prioritizing COVID-19 vaccination by both age and neighbourhood of residence is an effective strategy to minimize deaths, morbidity, and hospitalization. Targeting people living in NORCs in high-risk neighbourhoods for early vaccination is a practical application of that strategy, which will also address barriers to vaccination in this population.

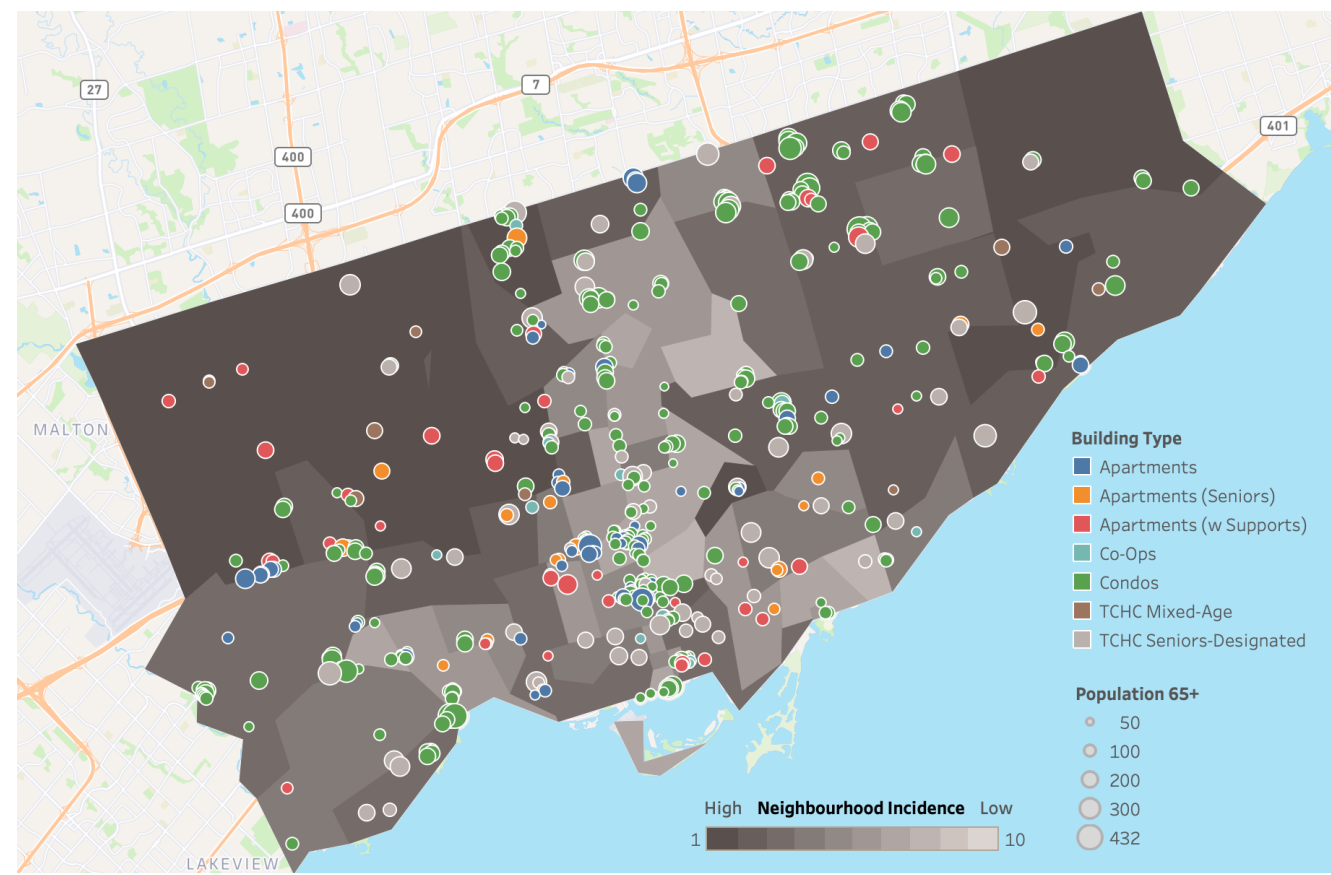

Figure 1. Location and Population Size of 489 Naturally Occurring Retirement Communities (NORCs) in the City of Toronto, by Building Type and Neighbourhood Incidence of SARS-CoV-2 from January 23, 2020 to January 16, 2021 Shaded map showing Toronto neighbourhoods ranked in 10\% increments of COVID-19 risk by the cumulative incidence of SARS-CoV-2 infections among Ontario neighourhoods from Jan 23, 2020 and Jan 16, 2021. Neighbourhoods are defined by the first three characters of a resident's postal code, known as "forward sortation area". Group 1 includes neighbourhoods with the highest cumulative incidence of SARS-CoV-2 infection, whereas group 10 includes neighbourhoods with the lowest cumulative incidence of SARS-CoV-2 infection. The City of Toronto 
does not have any neighbourhoods in risk groups 9 or 10. The map is overlaid with 489 NORCs, defined as apartment, condo, co-op and social housing (Toronto Community Housing Corporation) buildings with at least 30 percent of their residents being 65 years of age and above, and with at least 50 older persons per building. Data for cumulative incidence of SARS-CoV-2 infections sourced from the Public Health Case and Contact Management Solution and other case management systems (CCM plus), extracted on January 16, 2021; data for demographics of NORCs sourced from the Registered Persons Database for fiscal year 2019/2020.

\section{Summary}

\section{Background}

Ontario's COVID-19 Science Advisory Table reported that prioritizing COVID-19 vaccination by both age and neighbourhood of residence could ensure that those at highest risk of SARS-CoV-2 infections, and COVID-19 hospitalizations, ICU admissions, and deaths will be among the first to receive vaccines. Accordingly, on March 5, 2021 Ontario's COVID-19 vaccine distribution plan was updated to focus on both, age and neighbourhood risk, by including people who live in "hot spots." One option to consider is vaccination programs for naturally occurring retirement communities (NORCs), defined as apartment, condo, co-op and social housing buildings with at least 30 percent of their residents aged 65 years or above, and with at least 50 older people per building.

\section{Question}

Where are NORCs located in the City of Toronto and what is the age distribution of their inhabitants?

How many older adults live in NORCs in the City of Toronto that are located in high SARS-CoV-2 incidence neighbourhoods where targeted vaccination could reduce SARS-CoV-2 cases, COVID-19 hospitalizations and deaths?

\section{Findings}

In Toronto there are 489 residential buildings that can be characterized as NORCs. They are home to 70,013 older adults 65 years of age and above, including 30,346 aged 80 years and above. Of these, 256 NORCs are located in high SARS-CoV-2 incidence neighbourhoods, accounting for 40,955 adults 65 years of age and above, including 18,144 older adults 80 years of age and above.

\section{Interpretation}

Targeting NORCs through mobile on-site vaccination could be an efficient and equitable approach to protecting those at highest risk of COVID-19 hospitalization, morbidity and mortality. Such an approach is also age-friendly, as it is less complicated and physically taxing for frail or homebound older adults who might otherwise face challenges with booking appointments, travelling to and from mass vaccination clinics, and have difficulty waiting in lines.

\section{Background}

Ontario's COVID-19 Science Advisory Table reported that prioritizing COVID-19 vaccination by both age and neighbourhood of residence could ensure that those at highest risk of SARS-CoV-2 infections, and COVID-19 hospitalizations, ICU admissions, and deaths will be among the first to receive vaccines. ${ }^{1}$ Such a vaccination strategy was projected to prevent 3,767 SARS-CoV-2 cases, and 702 hospitalizations, 145 ICU admissions, and 168 deaths due to COVID-19 as compared to a strategy that prioritizes vaccination based on age alone. ${ }^{1}$ 
On March 5, 2021, the Ontario government updated its COVID-19 vaccine distribution plan to include a focus on age and neighbourhood risk, including people who live in high risk neighbourhoods or "hot spots". ${ }^{2}$ Select public health units (PHUs) will be allocated up to 920,000 COVID-19 vaccine doses during Phase 2 to vaccinate Ontarians residing in neighbourhoods with high SARS-CoV-2 incidence. ${ }^{3}$ These PHUs will then determine optimal strategies to distribute vaccines to their hot spots based on local demographics.

Naturally occurring retirement communities (NORCs) are apartment, condo, co-op and social housing buildings with at least 30 percent of their residents being 65 years of age and above, and with at least 50 older persons per building. They differ from long-term care (LTC) and retirement homes which are purpose-built for older adults. ${ }^{4}$ Since many NORCs may be found in high risk neighbourhoods, and since the majority of their inhabitants are older adults, they represent a promising example of priority overlap for Ontario's COVID-19 vaccination program.

In addition, many NORC residents may face challenges with booking appointments and travelling to and from mass vaccination clinics, and as such they could be targets for mobile on-site vaccination much like long-term care, retirement homes and other congregate care settings during Phase 1 of Ontario's COVID-19 vaccine distribution plan. ${ }^{5}$

Since NORCs are high-density residential settings, they allow for a practical, efficient and targeted mobile approach to vaccinating those at high risk of SARS-CoV-2 infection, and COVID-19 hospitalization and death. However, unlike long-term care and retirement homes, NORCs are ill-defined, their locations are more difficult to pinpoint, and the socio-demographic characteristics of their residents are not well understood.

\section{Question}

Where are NORCs located in the City of Toronto and what is the age distribution of their inhabitants?

How many older adults live in NORCs in the City of Toronto that are located in high SARS-CoV-2 incidence neighbourhoods where targeted vaccination could reduce SARS-CoV-2 cases, and COVID-19 hospitalizations and deaths?

\section{Findings}

We identified 489 NORCs in the City of Toronto, which are home to 70,013 older adults 65 years of age and above, including 30,346 aged 80 years and above. The majority of these are condominiums (283), followed by various types of apartments including those with supports (115), social housing (74) and co-ops (17). These buildings were mapped on an online interactive tool containing the age distribution of their residents:

\footnotetext{
Interactive online tool mapping NORCs in the City of Toronto
} 


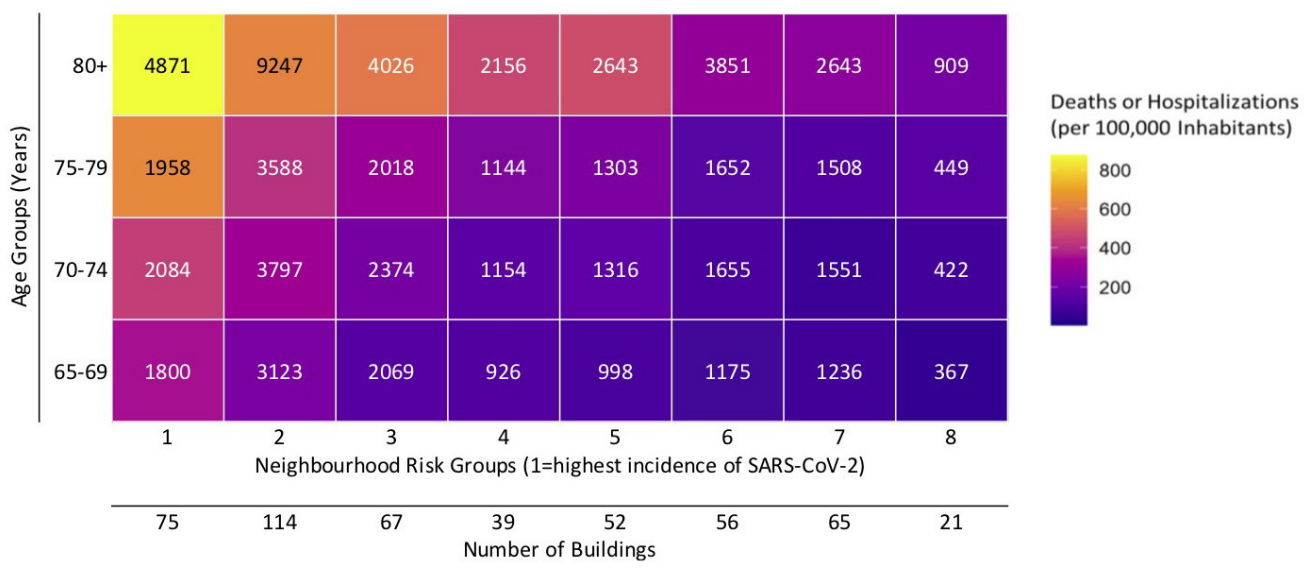

Figure 2. Number of Older Adults Living in Naturally Occurring Retirement Communities (NORCs) in the City of Toronto, by Age and Neighbourhood Incidence of SARS-CoV-2 from January 23, 2020 to January 16, 2021

The number of older adults residing in Toronto's 489 NORCs by age and neighbourhood risk group. Neighbourhood risk group 1 includes neighbourhoods ranked in the top 10\% of cumulative SARS-CoV-2 incidence among all Ontario neighbourhoods between January 23, 2020 and January 16, 2021, group 10 includes neighbourhoods ranked in the bottom 10\% of cumulative SARS-CoV-2 incidence; the City of Toronto does not have any neighbourhoods in risk groups 9 or 10. The colour gradient depicts the incidence of COVID-19 deaths or hospitalizations per 100,000 inhabitants by age and neighbourhood among all Torontorians living outside of LTC and retirement homes from January 23, 2020 to January 16, 2021. The numbers below the neighbourhood risk group at the bottom of the figure represent the number of NORCs located in each neighbourhood risk group. There are 70,013 older adults living in Toronto's 489 NORCs. A total of 40,955 (58.5\%) live in 256 NORCs (52.4\%) located in Ontario's top 30\% of neighbourhoods with the highest cumulative SARS-CoV-2 incidence. Data for cumulative incidence of SARS-CoV-2 infections sourced from the Public Health Case and Contact Management Solution and other case management systems (CCM plus), extracted on January 16, 2021; data for demographics of NORCs sourced from the Registered Persons Database (RPDB) for fiscal year 2019/2020.

There are 256 out of 489 NORCs (52.4\%) in Toronto located in Ontario's top 30\% of neighbourhoods with the highest cumulative SARS-CoV-2 incidence (Figure 2). ${ }^{1}$ These 256 NORCs situated in high SARS-CoV-2 incidence neighhourhoods are home to 40,955 adults aged 65 years or above, which corresponds to $58.5 \%$ of all older adult residents living in Toronto's NORCs; 18,144 are aged 80 years or above. Many of these NORCs are located in Toronto's northwest and east (Scarborough) regions (Figure 3).

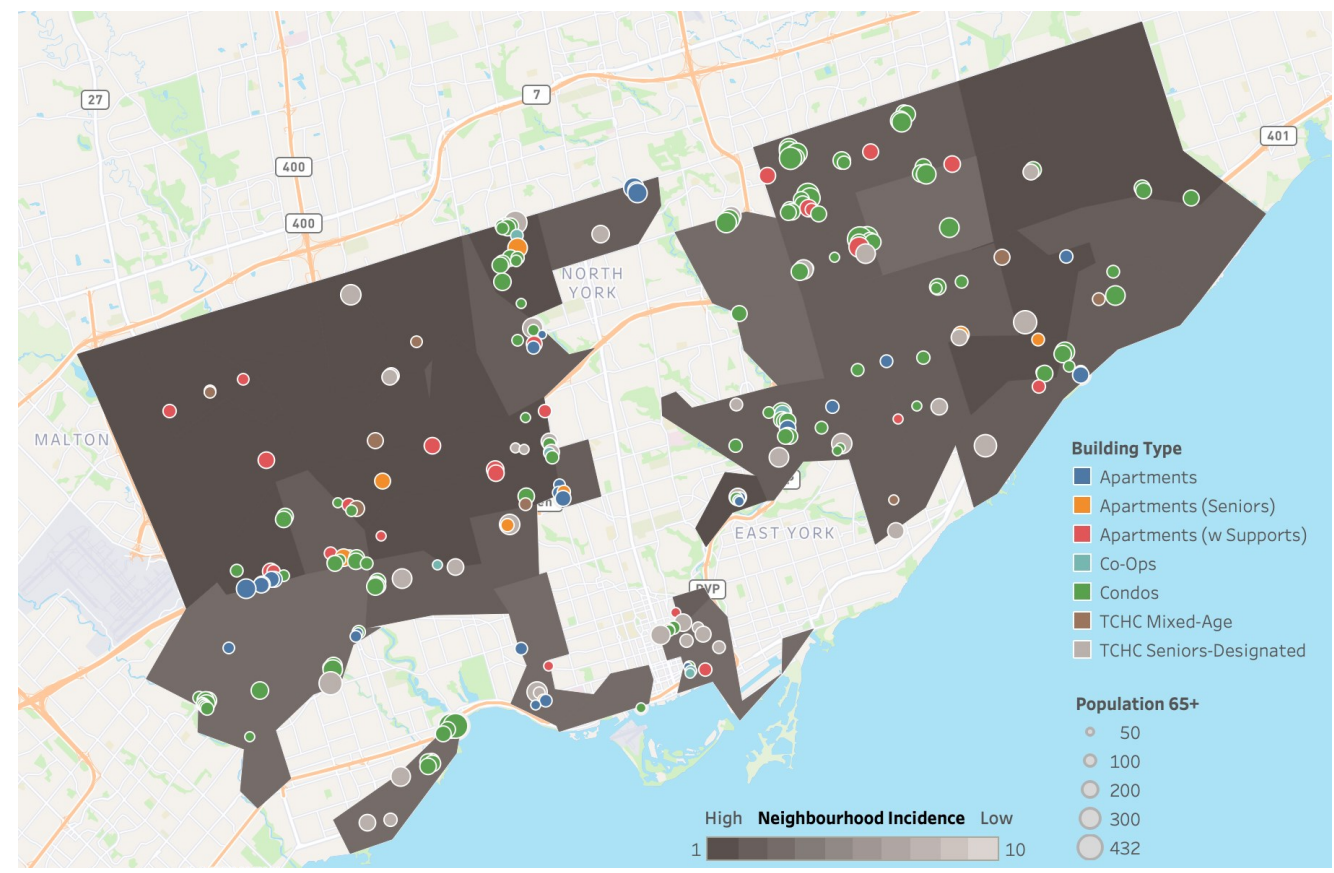

Figure 3. Location, Building Type and Population Size of 256 Naturally Occurring Retirement Communities (NORCs) Located in 48 Toronto Neighbourhoods Ranked in the Top 30\% of Cumulative SARS-CoV-2 Incidence Among all Ontario Neighbourhoods Between January 23, 2020 and January 16, 2021

Shaded map of 256 NORC located in 48 Toronto neighbourhoods ranked in the top 30\% of cumulative SARS-CoV-2 incidence among all Ontario neighbourhoods (neighbourhood risk groups 1-3) between January 23, 2020 and 
January 16, 2021. Neighbourhood risk groups 4 to 8 are not shaded, Toronto does not have neighbourhoods in risk groups 9 and 10. Neighbourhoods are defined by the first three characters of a resident's postal code, known as "forward sortation area". The map is overlaid with 256 NORCs defined as apartment, condo, co-op and social housing (Toronto Community Housing Corporation) buildings with at least 30 percent of their residents aged 65 years or above, and with at least 50 older people per building. Data for cumulative incidence of SARS-CoV-2 infections sourced from the Public Health Case and Contact Management Solution and other case management systems (CCM plus), extracted on January 16, 2021; data for demographics of NORCs sourced from the Registered Persons Database for fiscal year 2019/2020.

\section{Interpretation}

Targeting NORCs through mobile on-site vaccination could be an efficient and equitable approach to protecting those at highest risk of COVID-19 hospitalization, morbidity and mortality. It can help ensure many older adults at high risk of SARSCoV-2 infection and poor COVID-19 outcomes are vaccinated as soon as possible. The strategy also has the additional benefit of removing potential barriers for individuals with inadequate computer proficiency or unfamiliarity with on-line booking systems, limited fluency in English or French, or those with cognitive or sensory impairment. The mobile-based strategy will also be less physically taxing for many frail or homebound older adults who might otherwise face challenges with travelling to and from mass vaccination clinics, and have difficulty waiting in lines.

The Toronto-based analysis presented here relied on readily-available open data, and is therefore replicable across the province. Our data, along with the mapping tool created, offer a way to systematically approach scale-up of mobile vaccination in NORCs, with an eye towards ensuring more rapid and equitable access to COVID19 vaccinations among older adults.

Similar analyses have yet to be completed for other municipalities or PHUs, but it is anticipated that the number of NORCs and their residents in Toronto far exceed that of other Ontario jurisdictions. Census data showed that in the City of Toronto, there were 142,990 older adults aged 65 years or above (36\% of older Torontonians) living in dwellings 5 storeys or taller. ${ }^{6,7}$ For comparison, the PHU with the next highest number of older adults 65 years of age and above living in dwellings 5 storeys or taller is Peel, with 29,090 (17\%), followed by Ottawa with $25,410(19 \%)$, York with 19,950 (13\%), and Hamilton with 14,290 (16\%).

Pilot mobile vaccination programs in several NORC apartment buildings for influenza vaccinations in fall 2020 by the Mid-West Toronto Ontario Health Team and University Health Network OpenLab (Huynh T, personal communication) as well as for COVID-19 vaccines in February 2021 by the University Health Network and Michael Garron Hospital have already demonstrated the practicality and feasibility of this approach. ${ }^{8}$

There are several practical considerations when providing mobile on-site vaccination to NORCs. First, unlike long-term care and retirement homes, many NORCs have no existing relationship with the health care system. As such, outreach efforts to facility management and residents are necessary to plan a vaccination initiative. These efforts will be most effective if they involve trusted and familiar community members, which will maximize vaccine uptake. For example, in Toronto this could include the hundreds of "neighbourhood ambassadors" the city is hiring to promote vaccines in their communities. ${ }^{9}$

Second, mobile vaccination will require a supply of COVID-19 vaccines with less stringent cold chain and handling requirements. Third, NORCs may have physical infrastructure limitations such as slow or broken elevators as well as the absence of common rooms where an on-site clinic could be set up. ${ }^{10,11}$ Accordingly, door-todoor vaccination should be considered, as was done during Mid-West Toronto 
Ontario Health Team and UHN OpenLab's influenza vaccination efforts in the fall of 2020 (Figure 4). Additionally, collaboration with health care providers such as community paramedics who have experience and are familiar with the NORC environments will be beneficial.

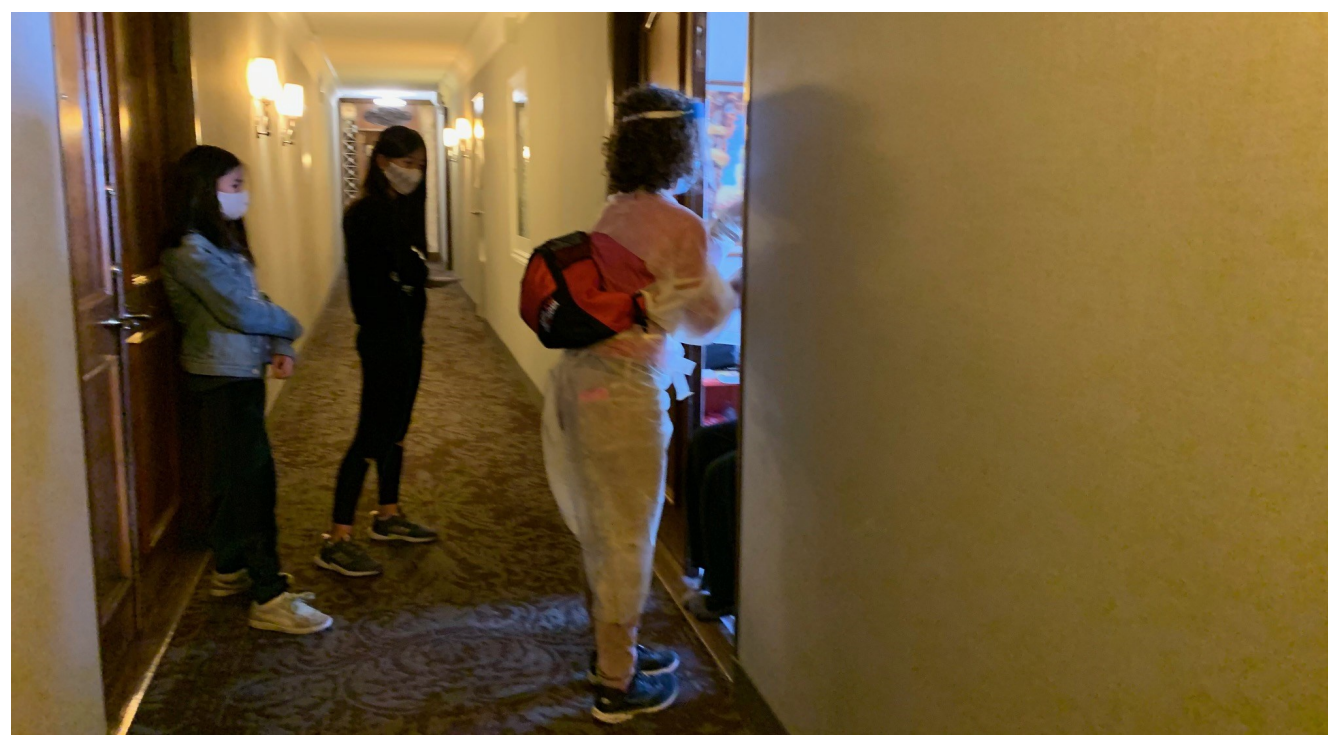

Figure 4. Mid-West Toronto Ontario Health Team and UHN OpenLab completing door-to-door influenza vaccination in a NORC in November 2020

Fourth, once inside a NORC, the mobile on-site vaccination team needs to be coordinated and divided into smaller units responsible for different floors, with each unit consisting of vaccinator(s) and volunteers/other staff who greet the residents, confirm informed consent, vaccinate, collect data and monitor the resident for any immediate adverse reactions to the vaccine.

Fifth, mobile on-site vaccination teams should be aware of potential barriers to vaccine uptake. This includes minimizing language barriers, by ensuring that mobile teams are equipped to communicate in the dominant languages of the residents. Residents also need to be supported in completing the current lengthy consent forms, which ideally could be provided to them in advance.

Sixth, when vaccinating a NORC, particularly in a high-risk community, the entire building should be considered for vaccination (not just older adults), since many are mix-aged communities where residents are heavily intertwined and interactions between people within buildings are high.

The proposed approach to vaccinating NORCs could also be applied to other communities living in large tower blocks and buildings ("vertical" communities) or other high-density living environments situated in high SARS-CoV-2 risk neighbourhoods, which would further advance uptake of COVID-19 vaccines in Ontario's hot spots.

Beyond the COVID-19 pandemic, NORCs represent an important opportunity to rethink and redesign how older adults age in their own homes. Currently available housing and care options can perpetuate the sense of loneliness, social and physical isolation, and result in institutionalization as people age. COVID-19 has exposed these shortcomings in a stark and dramatic way. When many older adults live right next door to one another, separated by just a wall or a ceiling, this opens up the possibility of re-designing services to make use of these natural densities and deliver a more expansive notion of care, on site. ${ }^{9}$ In so doing, older adults may be able to remain living independently in their own homes longer, taking pressures off downstream institutional care options. Indeed, most older adults want to remain in 
their homes for as long as possible. Optimizing NORCs enable older adults to age in their own homes with choice and dignity. The approach to COVID-19 vaccination in NORCs may provide a roadmap for a broader re-imagining of service delivery that supports aging in place.

\section{Methods Used for This Science Brief}

We retrieved the age distribution for all residents within each of the City of Toronto's 44,935 postal codes from the Registered Persons Database for fiscal year 2019/2020. We then identified 853 postal codes with at least 30 percent of their residents being 65 years of age and older, and with at least 50 older persons per postal code. Postal codes corresponding with high-density buildings were identified by matching them with those contained in geospatial datasets for long-term care and retirement homes, ${ }^{12}$ co-ops, ${ }^{13}$ and social housing. ${ }^{14,15}$ Addresses were also obtained through this process. The remaining building types (apartments and condos) were manually identified through Google searches of the postal codes. Each building was then assigned to a COVID-19 risk group based on the cumulative SARSCoV-2 incidence in its forward sortation area (FSA; the first three characters of the building's postal code) from the beginning of the COVID-19 pandemic (January 23, 2020) until January 16, 2021, with risk group 1 having the highest incidence and 10 the lowest incidence, by provincial decile. Geocoding was then completed to assign longitude and latitude to each building based on address. Finally, each building was mapped using Tableau software. ${ }^{16}$

\section{Author Contributions}

TH conceived the Science Brief and wrote the first draft. KAB and PJ provided data on neighbourhood risk groups. All authors contributed to the conception of the Science Brief, revised it for important intellectual content, and approved the final version.

The authors would like to thank the team at UHN OpenLab for developing and making public the interactive map underpinning this Science Brief.

\section{References}

1. Brown KA, Stall NM, Joh E, et al. COVID-19 Vaccination Strategy for Ontario Using Age and Neighbourhood-Based Prioritization. Science Briefs of the Ontario COVID-19 Science Advisory Table; 2021. Accessed March 6, 2021. https://covid19sciencetable.ca/sciencebrief/covid-19-vaccination-strategy-for-ontario-using-ageand-neighbourhood-based-prioritization

2. Government of Ontario. Ontario ready to rollout phase two of COVID-19 vaccine distribution plan. Published March 5, 2021. Accessed March 6, 2021. https:// news.ontario.ca/en/release/60568/ontario-ready-to-rollout-phase-two-of-covid-19vaccine-distribution-plan-1

3. Government of Ontario. Populations eligible for phase two COVID-19 vaccination. Published March 5, 2021. Accessed March 7, 2021. https://news.ontario.ca/en/ backgrounder/60570/populations-eligible-for-phase-two-covid-19-vaccination

4. Hunt ME, Gunter-Hunt G. Naturally Occurring Retirement Communities. J Hous Elder. 1986;3(3-4):3-22. https://doi.org/10.1300/J081V03N03_02

5. Government of Ontario. COVID-19 vaccines for Ontario. COVID-19 in Ontario. Published 2021. Accessed March 6, 2021. https://covid-19.ontario.ca/covid-19vaccines-ontario 
6. Huynh T. Aging in the vertical city. The Local. Published online June 13, 2018. Accessed March 6, 2021. https://thelocal.to/aging-in-the-vertical-citycbda1cb4f351/

7. Government of Canada. 2016 census of Canada: data tables - structural type of dwelling (10), age (20) and sex (3) for the population in occupied private dwellings of Canada, provinces and territories, census divisions and census subdivisions, 2016 Census - 100\% Data. Published May 3, 2017. Accessed March 9, 2021. https:// www12.statcan.gc.ca/census-recensement/2016/dp-pd/dt-td/Rp-eng.cfm? LANG $=E \& A P A T H=3 \& D E T A I L=0 \& D I M=0 \& F L=A \& F R E E=0 \& G C=0 \& G I D=0 \& G K=0 \& G R P=1$ $\& P I D=109534 \& P R I D=10 \& P T Y P E=109445 \& S=0 \& S H O W A L L=0 \& S U B=0 \& T e m p o r a l=201$ $6 \&$ THEME $=116 \& V I D=0 \& V N A M E E=\& V N A M E F=$

8. Warren M. New pilot program brings COVID-19 vaccines - and hope - to the door for hundreds of Toronto seniors. Toronto Star. https://www.thestar.com/ news/gta/2021/02/23/new-pilot-program-brings-covid-19-vaccines-and-hope-tothe-door-for-hundreds-of-toronto-seniors.html. Published February 23, 2021. Accessed March 7, 2021.

9. City of Toronto. City of Toronto provides updated details of city COVID-19 vaccination program and collaboration with health sector partners. City of Toronto. Published March 1, 2021. https://www.toronto.ca/news/city-of-toronto-providesupdated-details-of-city-covid-19-vaccination-program-and-collaboration-with-health -sector-partners/

10. MacDonnell S, Robinson J, Mikadze V, McDonough L, Meisner A. Vertical Poverty: Poverty by Postal Code 2. United Way Toronto; 2011. https:// www.unitedwaygt.org/document.doc?id=89

11. University Health Network OpenLab. Vertical aging: the future of aging in place in urban Canada, social spaces (No. 3). Accessed March 8, 2021. http:// verticalaging.uhnopenlab.ca/social-spaces/

12. Ontario GeoHub. Ministry of health service provider locations. Published April 3, 2020. Accessed March 7, 2021. https://geohub.lio.gov.on.ca/datasets/ministry-ofhealth-service-provider-locations

13. Co-operative Housing Federation of Toronto. Find a co-op. Accessed March 7, 2021. https://co-ophousingtoronto.coop/resources/find-a-coop/

14. City of Toronto. Address points (municipal) - Toronto one address repository. Published March 15, 2021. https://ckan0.cf.opendata.inter.prod-toronto.ca/ne/ dataset/address-points-municipal-toronto-one-address-repository

15. Toronto Community Housing Corporation. Our housing. Published 2021. Accessed March 7, 2021. https://www.torontohousing.ca/our-housing

16. University Health Network OpenLab. Apartment, condo, co-op, and social housing buildings in Toronto with at least $30 \%$ of residents $65+$ and at least 50 older adults. Tableau Software. Published March 2, 2021. Accessed March 16, 2021. https://public.tableau.com/views/High-DensitySeniorsBuildingsinToronto/ Dashboard1?\%3Aembed=y\&\%3AshowVizHome=no\&\%3Adisplay_count=y\&\% 3Adisplay_static_image $=y \& \% 3 A$ bootstrapWhenNotified $=$ true $\& \%$ 3Alanguage=en \&:embed=y\&:showVizHome=n\&:apilD=host0\#navType=0\&navSrc= 